\title{
The second largest eigenvalues of some Cayley graphs on alternating groups*
}

\author{
Xueyi Huang, Qiongxiang Huang ${ }^{\dagger}$ \\ College of Mathematics and Systems Science, Xinjiang University, Urumqi, Xinjiang 830046, P. R. China
}

\begin{abstract}
Let $A_{n}$ denote the alternating group of degree $n$ with $n \geq 3$. The alternating group graph $A G_{n}$, extended alternating group graph $E A G_{n}$ and complete alternating group graph $C A G_{n}$ are the Cayley graphs $\operatorname{Cay}\left(A_{n}, T_{1}\right), \operatorname{Cay}\left(A_{n}, T_{2}\right)$ and $\operatorname{Cay}\left(A_{n}, T_{3}\right)$, respectively, where $T_{1}=\{(1,2, i),(1, i, 2) \mid 3 \leq i \leq n\}, T_{2}=$ $\{(1, i, j),(1, j, i) \mid 2 \leq i<j \leq n\}$ and $T_{3}=\{(i, j, k),(i, k, j) \mid 1 \leq i<j<k \leq n\}$. In this paper, we determine the second largest eigenvalues of $A G_{n}, E A G_{n}$ and $C A G_{n}$.
\end{abstract}

Keywords: Alternating group graph; Cayley graph; Second largest eigenvalue

AMS Classification: 05C50

\section{Introduction}

Let $G=(V(G), E(G))$ be a simple undirected graph of order $n$. The adjacency matrix of $G$, denoted by $A(G)$, is the $n \times n$ matrix with entries $a_{u v}=1$ if $\{u, v\} \in$ $E(G)$ and $a_{u v}=0$ otherwise. The eigenvalues of $A(G)$ are denoted by $\lambda_{1}(G) \geq$ $\lambda_{2}(G) \geq \cdots \geq \lambda_{n}(G)$, which are also called the eigenvalues of $G$.

For $v \in V(G)$, we denote by $N(v)=\{u \in V(G) \mid\{u, v\} \in E(G)\}$ and $d(v)=$ $|N(v)|$ the neighborhood and degree of $v$, respectively. Let $D(G)=\operatorname{diag}(d(v) \mid$ $v \in V(G)$ ) denote the diagonal degree matrix of $G$. The Laplacian matrix of $G$ is defined as $L(G)=D(G)-A(G)$, which is positive semi-definite and always has 0 as its eigenvalue. So the eigenvalues of $L(G)$ can be arranged as $\mu_{1}(G) \geq \mu_{2}(G) \geq \cdots \geq$ $\mu_{n-1}(G) \geq \mu_{n}(G)=0$. Furthermore, $\mu_{n-1}(G)>0$ if and only if $G$ is connected, and for connected non-complete graphs we know that $0<\mu_{n-1}(G) \leq \kappa(G) \leq \kappa^{\prime}(G) \leq$ $\delta(G)$, where $\kappa(G), \kappa^{\prime}(G)$ and $\delta(G)$ denote the vertex connectivity, edge connectivity and minimum degree of $G$, respectively (cf. [9], Corollary 7.4.6). For this reason, $\mu_{n-1}(G)$ is called the algebraic connectivity of $G$. Clearly, if $G$ is $r$-regular then $\mu_{n-1}(G)=r-\lambda_{2}(G)$.

*This work is supported by the National Natural Science Foundation of China (Grant nos. 11531011, 11671344).

${ }^{\dagger}$ Corresponding author.

E-mail address: huangxymath@gmail.com (X. Huang), huangqx@xju.edu.cn (Q. Huang). 
For a finite group $\Gamma$, and a subset $T \subseteq \Gamma \backslash\{e\}$ ( $e$ is the identity element of $\Gamma$ ) such that $T=T^{-1}$, the Cayley graph $\operatorname{Cay}(\Gamma, T)$ on $\Gamma$ with respect to $T$ is defined as the undirected graph with vertex set $\Gamma$ and edge set $\{\{\gamma, t \gamma\} \mid \gamma \in \Gamma, t \in T\}$. Clearly, $\operatorname{Cay}(\Gamma, T)$ is a $|T|$-regular graph which is connected if and only if $T$ is a generating subset of $\Gamma$.

Let $S_{n}$ be the symmetric group of degree $n \geq 3$ (for $\sigma, \tau \in S_{n}$, the product $\sigma \tau$ means that we first apply permutation $\sigma$ and then apply permutation $\tau$, and $\tau_{i}$ represents the image of $i$ under the permuation $\tau$ ), and let $T$ be a set of transpositions in $S_{n}$. The transposition graph $\operatorname{Tra}(T)$ of $T$ is defined as the graph with vertex set $\{1,2, \ldots, n\}$ and with an edge connecting two vertices $i$ and $j$ if and only if $(i, j) \in T$. It is known that $T$ can generate $S_{n}$ if and only if $\operatorname{Tra}(T)$ is connected [13]. Around 1992, Aldous [1] (see also [4,12]) conjectured that if $\operatorname{Tra}(T)$ is connected then the second largest eigenvalue of $\operatorname{Cay}\left(S_{n}, T\right)$ is equal to $|T|-\mu_{n-1}(\operatorname{Tra}(T))$, that is, $\operatorname{Cay}\left(S_{n}, T\right)$ and $\operatorname{Tra}(T)$ share the same algebraic connectivity. Before 1992, Flatto et al. [11] and Diaconis and Shahshahani [10] had confirmed the conjecture for $\operatorname{Tra}(T)=K_{1, n-1}$ and $\operatorname{Tra}(T)=K_{n}$, respectively. In 2000, Friedman [12] proved that if $\operatorname{Tra}(T)$ is a tree $(|T|=n-1)$ then the second largest eigenvalue of $\operatorname{Cay}\left(S_{n}, T\right)$ is at least $n-2$, and exactly equal to $n-2$ if and only if $\operatorname{Tra}(T)=K_{1, n-1}$. In 2010, Cesi [4] confirmed Aldous' conjecture when Tra $(T)$ is a complete multipartite graph. Almost at the same time, Caputo et al. [2] completely solved the conjecture for all connected Tra $(T)$. For other Cayley graphs on symmetric groups, Cesi [3] proved that the second largest eigenvalue of the pancake graph $\mathcal{P}_{n}=\operatorname{Cay}\left(S_{n}, S\right)$ $(S=\{(1, i)(2, i-1) \cdots \mid 2 \leq i \leq n\})$ is equal to $n-2$; and very recently, Chung and Tobin [8] determined the second largest eigenvalues of the reversal graph $R_{n}=$ $\operatorname{Cay}\left(S_{n}, S\right)(S=\{(i, j)(i+1, j-1) \cdots \mid 1 \leq i<j \leq n\})$ and a family of graphs that generalize the pancake graph. Their method is very imaginative, which depends on finding enough equitable partitions of $R_{n}$ and decomposing the edge set $E\left(R_{n}\right)$ to that of $\mathcal{P}_{n}$ and $n$ copies of $R_{n-1}$.

Now we consider the Cayley graphs on alternating group $A_{n}(n \geq 3)$. Let $T_{1}=\{(1,2, i),(1, i, 2) \mid 3 \leq i \leq n\}, T_{2}=\{(1, i, j),(1, j, i) \mid 2 \leq i<j \leq n\}$ and $T_{3}=\{(i, j, k),(i, k, j) \mid 1 \leq i<j<k \leq n\}$. The alternating group graph $A G_{n}$, extended alternating group graph $E A G_{n}$ and complete alternating group graph $C A G_{n}$ are defined as the Cayley graphs $\operatorname{Cay}\left(A_{n}, T_{1}\right), \operatorname{Cay}\left(A_{n}, T_{2}\right)$ and $\operatorname{Cay}\left(A_{n}, T_{3}\right)$, respectively. Since $T_{1} \subseteq T_{2} \subseteq T_{3}, A G_{n}$ is a spanning subgraph of $E A G_{n}$ while $E A G_{n}$ is also a spanning subgraph of $C A G_{n}$, and all these graphs are connected because $T_{1}$ can generate $A_{n}$ (cf. [16], p. 298). In [15], Jwo et al. introduced the alternating group graph $A G_{n}$ as an interconnection network topology for computing systems, and they also showed that $A G_{n}$ is 2-transitive, Hamiltonian and of diameter $\lfloor 3(n-2) / 2\rfloor$. Following this paper, $A G_{n}$ was extensively studied over decades. For example, Chang and Yang [5,6] investigated the panconnectivity, Hamiltonianconnectivity and fault-tolerant Hamiltonicity of $A G_{n}$. Zhou [17] determined the full automorphism group of $A G_{n}$. In addition, the complete alternating group graph $C A G_{n}$ can be viewed as one of the two isomorphic connected components of the $(n-3)$-point fixing graph $\mathcal{F}(n, n-3)$ defined in [7], which has only integral eigenvalues (cf. [7], Corollary 1.2). Recently, the full automorphism group of $C A G_{n}$ was determined by Huang and Huang [14]. 
Inspired by the work of Chung and Tobin [8], in the present paper, we determine the second largest eigenvalues of $A G_{n}, E A G_{n}$ and $C A G_{n}$, which are equal to $2 n-6$ (for $n \geq 4), n^{2}-5 n+5$ and $\frac{1}{3} n(n-2)(n-4)$, respectively.

The paper is organized as follows. In Section 2, we obtain the second largest eigenvalue of $A G_{n}$ by induction on $n$. In Section 3, we focus on determining the second largest eigenvalue of $C A G_{n}$. In order to achieve this goal by induction on $n$, we decompose the edge set of $C A G_{n}$ into that of $E A G_{n}$ and $n$ copies of $C A G_{n-1}$, and use $\lambda_{2}\left(E A G_{n}\right)+\lambda_{2}\left(C A G_{n-1}\right)$ to bound $\lambda_{2}\left(C A G_{n}\right)$. Thus we have to determine $\lambda_{2}\left(E A G_{n}\right)$, and for this reason, we also decompose the edge set of $E A G_{n}$ into that of $A G_{n}$ and $n$ copies of $E A G_{n-1}$, and use $\lambda_{2}\left(A G_{n}\right)+\lambda_{2}\left(E A G_{n-1}\right)$ to bound $\lambda_{2}\left(E A G_{n}\right)$ as $\lambda_{2}\left(A G_{n}\right)$ has been determined in Section 2.

\section{The second largest eigenvalue of $A G_{n}$}

In this section, we focus on determining the second largest eigenvalue of $A G_{n}$. In order to obtain enough information about the eigenvalues of $A G_{n}$, we need a well known result on equitable partition.

Let $G$ be a graph of order $n$, and let $\Pi: V(G)=V_{1} \cup V_{2} \cup \cdots \cup V_{k}$ be a vertex partition of $G$. The characteristic matrix $\chi_{\Pi}$ of $\Pi$ is the $n \times k$ matrix whose columns are the character vectors of $V_{1}, \ldots, V_{k}$. The partition $\Pi$ is called an equitable partition of $G$ if, for any $v \in V_{i},\left|N(v) \cap V_{j}\right|=b_{i j}$ is a constant only dependent on $i, j(1 \leq i, j \leq k)$. The matrix $B_{\Pi}=\left(b_{i j}\right)_{k \times k}$ is called the divisor matrix of $G$ with respect to $\Pi$.

Lemma 2.1 ( $[13])$. Let $G$ be a graph with adjacency matrix $A(G)$, and let $\Pi$ : $V(G)=V_{1} \cup V_{2} \cup \cdots \cup V_{k}$ be an equitable partition of $G$ with divisor matrix $B_{\Pi}$. Then each eigenvalue of $B_{\Pi}$ is also an eigenvalue of $A(G)$. Furthermore, $A(G)$ has the following two kinds of eigenvectors:

(i) the eigenvectors in the column space of $\chi_{\Pi}$, and the corresponding eigenvalues coincide with the eigenvalues of $B_{\Pi}$;

(ii) the eigenvectors orthogonal to the columns of $\chi_{\Pi}$, i.e., those eigenvectors that sum to zero on each block $V_{i}$ for $1 \leq i \leq k$.

Recall that $A G_{n}=\operatorname{Cay}\left(A_{n}, T_{1}\right)$, where $T_{1}=\{(1,2, i),(1, i, 2) \mid 3 \leq i \leq n\}$. Clearly, $A G_{n}$ is $(2 n-4)$-regular. If $n=3$, then $A G_{n}=K_{3}$, which has -1 as its second largest eigenvalue. Now suppose $n \geq 4$. For each fixed $i(1 \leq i \leq n)$, we define

$$
\begin{aligned}
& X(i)=\left\{\tau \in A_{n} \mid \tau_{n}=i\right\}, \\
& Y(i)=\left\{\tau \in A_{n} \mid \tau_{1}=i\right\}, \\
& Z(i)=\left\{\tau \in A_{n} \mid \tau_{2}=i\right\}, \\
& W(i)=\left\{\tau \in A_{n} \mid \tau_{1} \neq i, \tau_{2} \neq i, \tau_{n} \neq i\right\} .
\end{aligned}
$$

Now we verify that $\Pi: A_{n}=X(i) \cup Y(i) \cup Z(i) \cup W(i)$ is exactly an equitable partition of $A G_{n}$. For each $\tau \in X(i)$, we have $N(\tau) \cap X(i)=\{(1,2, k) \tau,(1, k, 2) \tau \mid 3 \leq k \leq$ $n-1\}, N(\tau) \cap Y(i)=\{(1, n, 2) \tau\}, N(\tau) \cap Z(i)=\{(1,2, n) \tau\}$ and $N(\tau) \cap W(i)=\emptyset$. 
This implies that each $\tau \in X(i)$ has exactly $2 n-6$ neighbors in $X(i)$, one neighbor in $Y(i)$, one neighbor in $Z(i)$ and no neighbors in $W(i)$. Similarly, for each $\tau \in Y(i)$, we have $N(\tau) \cap X(i)=\{(1,2, n) \tau\}, N(\tau) \cap Y(i)=\emptyset, N(\tau) \cap Z(i)=\{(1, k, 2) \tau$ | $3 \leq k \leq n\}$ and $N(\tau) \cap W(i)=\{(1,2, k) \tau \mid 3 \leq k \leq n-1\}$, and for each $\tau \in Z(i)$, we have $N(\tau) \cap X(i)=\{(1, n, 2) \tau\}, N(\tau) \cap Y(i)=\{(1,2, k) \tau \mid 3 \leq k \leq n\}$, $N(\tau) \cap Z(i)=\emptyset$ and $N(\tau) \cap W(i)=\{(1, k, 2) \tau \mid 3 \leq k \leq n-1\}$. For each $\tau \in W(i)$, we know that $\tau_{\ell}=i$ for some $3 \leq \ell \leq n-1$ because $\tau_{1}, \tau_{2}, \tau_{n} \neq i$. Then $N(\tau) \cap X(i)=\emptyset, N(\tau) \cap Y(i)=\{(1, \ell, 2) \tau\}, N(\tau) \cap Z(i)=\{(1,2, \ell) \tau\}$ and $N(\tau) \cap W(i)=\{(1,2, k) \tau,(1, k, 2) \tau \mid 3 \leq k \leq n, k \neq \ell\}$. Therefore, the divisor matrix of $A G_{n}$ with respect to $\Pi$ is equal to

$$
B_{\Pi}=\left[\begin{array}{cccc}
2 n-6 & 1 & 1 & 0 \\
1 & 0 & n-2 & n-3 \\
1 & n-2 & 0 & n-3 \\
0 & 1 & 1 & 2 n-6
\end{array}\right]
$$

By simple computation, we know that $B_{\Pi}$ has eigenvalues $2 n-4,2 n-6, n-4$ and $2-n$. Thus, by Lemma 2.1, we have

Lemma 2.2. Let $A G_{n}$ be the alternating group graph with $n \geq 4$. Then $A G_{n}$ has eigenvalues $2 n-4,2 n-6, n-4$ and $2-n$. Moreover, if $f$ is an eigenvector of $A G_{n}$ corresponding to any other eigenvalue, then for each $i \in\{1,2, \ldots, n\}$, we have

$$
\sum_{x \in X(i)} f(x)=\sum_{y \in Y(i)} f(y)=\sum_{z \in Z(i)} f(z)=\sum_{w \in W(i)} f(w)=0
$$

where $X(i), Y(i), Z(i)$ and $W(i)$ are defined in (1).

Now we are in a position to prove the main result of this section.

Theorem 2.3. Let $A G_{n}$ be the alternating group graph with $n \geq 4$. Then the two largest eigenvalues of $A G_{n}$ are $\lambda_{1}\left(A G_{n}\right)=2 n-4$ and $\lambda_{2}\left(A G_{n}\right)=2 n-6$.

Proof. Clearly, the largest eigenvalue of $A G_{n}$ is $\lambda_{1}\left(A G_{n}\right)=2 n-4$ because $A G_{n}$ is $(2 n-4)$-regular. We shall prove $\lambda_{2}\left(A G_{n}\right)=2 n-6$ by induction on $n$. If $n=4$, the result follows since all the distinct eigenvalues of $A G_{4}$ are 4, 2, 0 and -2 . Now suppose $n>4$, and assume that the result holds for $n-1$, that is, $\lambda_{2}\left(A G_{n-1}\right)=2(n-1)-6=2 n-8$.

Let $\lambda$ be an eigenvalue of $A G_{n}$ other than $2 n-4,2 n-6, n-4$ and $2-n$. It suffices to show $\lambda<2 n-6$. Take any fixed eigenvector $f$ of $A G_{n}$ corresponding to $\lambda$. We have the following claim.

Claim 1. There exists an $i$ such that

$$
2 \sum_{x \in X(i)} f(x)^{2} \geq \sum_{y \in Y(i)} f(y)^{2}+\sum_{z \in Z(i)} f(z)^{2}
$$

and

$$
\sum_{x \in X(i)} f(x)^{2}>0
$$


Proof. Notice that the vertex set of $A G_{n}$ can be partitioned in the following ways: $A_{n}=X(1) \cup X(2) \cup \cdots \cup X(n)=Y(1) \cup Y(2) \cup \cdots \cup Y(n)=Z(1) \cup Z(2) \cup \cdots \cup Z(n)$. Thus we have

$$
\sum_{j=1}^{n} \sum_{x \in X(j)} f(x)^{2}=\sum_{j=1}^{n} \sum_{y \in Y(j)} f(y)^{2}=\sum_{j=1}^{n} \sum_{z \in Z(j)} f(z)^{2}>0,
$$

and hence

$$
\sum_{j=1}^{n} 2 \sum_{x \in X(j)} f(x)^{2}=\sum_{j=1}^{n}\left(\sum_{y \in Y(j)} f(y)^{2}+\sum_{z \in Z(j)} f(z)^{2}\right)>0
$$

Therefore, there exists an index $i$ such that

$$
2 \sum_{x \in X(i)} f(x)^{2} \geq \sum_{y \in Y(i)} f(y)^{2}+\sum_{z \in Z(i)} f(z)^{2} .
$$

Let $I$ be the set of indices $i$ satisfying this inequality. Then we claim that there exists some $i \in I$ satisfying

$$
\sum_{x \in X(i)} f(x)^{2}>0
$$

because $f \neq 0$.

Now fix an $i$ satisfying the inequalities in Claim 1, and consider an arbitrary vertex $x \in X(i)$. As noted above, $x$ has $2 n-4$ neighbors in $A G_{n}$, in which $2 n-6$ neighbors in $X(i)$, one neighbor in $Y(i)$ and one neighbor in $Z(i)$. Observe that the induced subgraph of $A G_{n}$ on $X(i)$ is isomorphic to $A G_{n-1}=\operatorname{Cay}\left(A_{n-1}, T_{1}^{\prime}\right)$, where $T_{1}^{\prime}=\{(1,2, k),(1, k, 2) \mid 3 \leq k \leq n-1\}$. In fact, if $i=n$ then $A G_{n}[X(i)]$ is obviously isomorphic to $A G_{n-1}$ since $\tau_{n}=n$ for each $\tau \in X(n)$; if $i<n$, we define

$$
\begin{aligned}
& \phi: X(i) \longrightarrow A_{n-1} \\
& \tau=\left(\begin{array}{cccccccc}
1 & 2 & \cdots & l & \cdots & n-1 & n \\
\tau_{1} & \tau_{2} & \cdots & n & \cdots & \tau_{n-1} & i
\end{array}\right) \longmapsto\left(\begin{array}{cccccc}
1 & 2 & \cdots & l & \cdots & n-1 \\
\tau_{1} & \tau_{2} & \cdots & i & \cdots & \tau_{n-1}
\end{array}\right)
\end{aligned}
$$

It is easy to check that $\phi$ is one-to-one and onto. For any two distinct vertices $\tau=\left(\begin{array}{cccccccc}1 & 2 & \cdots & l & \cdots & n-1 & n \\ \tau_{1} & \tau_{2} & \cdots & n & \cdots & \tau_{n-1} & i\end{array}\right)$ and $\tau^{\prime}=\left(\begin{array}{ccccccc}1 & 2 & \cdots & m & \cdots & n-1 & n \\ \tau_{1}^{\prime} & \tau_{2}^{\prime} & \cdots & n & \cdots & \tau_{n-1}^{\prime} & i\end{array}\right)$ of $X(i)$, we have

$$
\begin{aligned}
& \tau^{\prime} \tau^{-1}=\left(\begin{array}{cccccccc}
1 & 2 & \cdots & m & \cdots & n-1 & n \\
\tau_{1}^{\prime} & \tau_{2}^{\prime} & \cdots & n & \cdots & \tau_{n-1}^{\prime} & i
\end{array}\right)\left(\begin{array}{ccccccc}
\tau_{1} & \tau_{2} & \cdots & n & \cdots & \tau_{n-1} & i \\
1 & 2 & \cdots & l & \cdots & n-1 & n
\end{array}\right) \\
& =\left(\begin{array}{ccccccc}
1 & 2 & \cdots & m & \cdots & n-1 & n \\
* & * & \cdots & l & \cdots & * & n
\end{array}\right) \\
& =\left(\begin{array}{cccccc}
1 & 2 & \cdots & m & \cdots & n-1 \\
* & * & \cdots & l & \cdots & *
\end{array}\right) \\
& =\left(\begin{array}{cccccc}
1 & 2 & \cdots & m & \cdots & n-1 \\
\tau_{1}^{\prime} & \tau_{2}^{\prime} & \cdots & i & \cdots & \tau_{n-1}^{\prime}
\end{array}\right)\left(\begin{array}{cccccc}
\tau_{1} & \tau_{2} & \cdots & i & \cdots & \tau_{n-1} \\
1 & 2 & \cdots & l & \cdots & n-1
\end{array}\right)
\end{aligned}
$$




$$
=\phi\left(\tau^{\prime}\right) \phi(\tau)^{-1}
$$

Then $\left\{\tau, \tau^{\prime}\right\} \in E\left(G\left[X_{i}\right]\right)$ if and only if $\tau^{\prime} \tau^{-1} \in T_{1}^{\prime}$, which is the case if and only if $\phi\left(\tau^{\prime}\right) \phi(\tau)^{-1} \in T_{1}^{\prime}$, which is the case if and only if $\left\{\phi(\tau), \phi\left(\tau^{\prime}\right)\right\} \in E\left(A G_{n-1}\right)$. Thus $\phi$ is exactly an isomorphism from $G[X(i)]$ to $A G_{n-1}$. Also note that the edges between $X(i)$ and $Y(i)$ (resp. $X(i)$ and $Z(i)$ ) form a matching. Let $x^{\prime}$ (resp. $x^{\prime \prime}$ ) be the unique neighbor of $x$ in $Y(i)$ (resp. $Z(i)$ ). By the eigenvalue-eigenvector equation, we have

$$
\lambda f(x)=\sum_{y \in N(x) \cap X(i)} f(y)+f\left(x^{\prime}\right)+f\left(x^{\prime \prime}\right)
$$

and further,

$$
\lambda f(x)^{2}=\sum_{y \in N(x) \cap X(i)} f(x) f(y)+f(x)\left(f\left(x^{\prime}\right)+f\left(x^{\prime \prime}\right)\right) .
$$

Summing both sides over $x \in X(i)$, we obtain

$$
\lambda \sum_{x \in X(i)} f(x)^{2}=\sum_{x \in X(i)} \sum_{y \in N(x) \cap X(i)} f(x) f(y)+\sum_{x \in X(i)} f(x)\left(f\left(x^{\prime}\right)+f\left(x^{\prime \prime}\right)\right),
$$

which gives that

$$
\lambda=\frac{\sum_{x \in X(i)} \sum_{y \in N(x) \cap X(i)} f(x) f(y)}{\sum_{x \in X(i)} f(x)^{2}}+\frac{\sum_{x \in X(i)} f(x)\left(f\left(x^{\prime}\right)+f\left(x^{\prime \prime}\right)\right)}{\sum_{x \in X(i)} f(x)^{2}} .
$$

Here we use the fact $\sum_{x \in X(i)} f(x)^{2}>0$ by Claim 1. Now we shall find upper bounds for the two terms in (2). Let $G_{1}=A G_{n}[X(i)]$. Then $G_{1} \cong A G_{n-1}$ as mentioned above. Set $g=\left.f\right|_{X(i)}$. We have $g \perp \mathbf{1}$ since $\sum_{x \in X(i)} f(x)=0$ according to Lemma 2.2 , where $\mathbf{1}$ is the all ones vector, which is also the eigenvector of $\lambda_{1}\left(G_{1}\right)$. Thus

$$
\begin{aligned}
\frac{\sum_{x \in X(i)} \sum_{y \in N(x) \cap X(i)} f(x) f(y)}{\sum_{x \in X(i)} f(x)^{2}} & =\frac{g^{T} A\left(G_{1}\right) g}{g^{T} g} \\
& \leq \max _{h \perp \mathbf{1}} \frac{h^{T} A\left(G_{1}\right) h}{h^{T} h} \\
& =\lambda_{2}\left(G_{1}\right) \\
& =\lambda_{2}\left(A G_{n-1}\right) \\
& =2 n-8 .
\end{aligned}
$$

Next consider the second term in (2). Recall that the edges between $X(i)$ and $Y(i)$, and $X(i)$ and $Z(i)$ form two matchings. Thus when $x$ range over the vertices of $X(i), x^{\prime}$ and $x^{\prime \prime}$ will range over the vertices of $Y(i)$ and $Z(i)$, respectively. Then

$$
\begin{aligned}
\frac{\sum_{x \in X(i)} f(x)\left(f\left(x^{\prime}\right)+f\left(x^{\prime \prime}\right)\right)}{\sum_{x \in X(i)} f(x)^{2}} & \leq \frac{\sqrt{\left(\sum_{x \in X(i)} f(x)^{2}\right)\left(\sum_{x \in X(i)}\left(f\left(x^{\prime}\right)+f\left(x^{\prime \prime}\right)\right)^{2}\right)}}{\sum_{x \in X(i)} f(x)^{2}} \\
& =\sqrt{\frac{\sum_{x \in X(i)}\left(f\left(x^{\prime}\right)+f\left(x^{\prime \prime}\right)\right)^{2}}{\sum_{x \in X(i)} f(x)^{2}}}
\end{aligned}
$$




$$
\begin{aligned}
& \leq \sqrt{\frac{\sum_{x \in X(i)} 2\left(f\left(x^{\prime}\right)^{2}+f\left(x^{\prime \prime}\right)^{2}\right)}{\sum_{x \in X(i)} f(x)^{2}}} \\
& =\sqrt{\frac{\left.2\left(\sum_{y \in Y(i)} f(y)^{2}+\sum_{z \in Z(i)} f(z)^{2}\right)\right)}{\sum_{x \in X(i)} f(x)^{2}}} \\
& \leq \sqrt{\frac{2 \cdot 2 \sum_{x \in X(i)} f(x)^{2}}{\sum_{x \in X(i)} f(x)^{2}}} \\
& =2,
\end{aligned}
$$

where the first inequality follows from the Cauchy-Schwarz inequality, the second use the fact $(a+b)^{2} \leq 2\left(a^{2}+b^{2}\right)$ and the third follows from Claim 1 .

Combing the above two bounds with (2) yields

$$
\lambda \leq 2 n-8+2=2 n-6,
$$

which implies that there are no eigenvalues in $(2 n-6,2 n-4)$. Therefore, we have $\lambda_{2}\left(A G_{n}\right)=2 n-6$.

\section{The second largest eigenvalues of $E A G_{n}$ and $C A G_{n}$}

Recall that the complete alternating group graph is defined as $C A G_{n}=\operatorname{Cay}\left(A_{n}, T_{3}\right)$, where $T_{3}=\{(i, j, k),(i, k, j) \mid 1 \leq i<j<k \leq n\}$ is the set of all 3-cycles in $S_{n}$. In this section, we mainly focus on determining the second largest eigenvalue of $C A G_{n}$. The main idea is to find some suitable equitable partition so that we can decompose the edge set of $C A G_{n}$ into that of $n$ copies of $C A G_{n-1}$ and that of the extended alternating group graph $E A G_{n}=\operatorname{Cay}\left(A_{n}, T_{2}\right)$, where $T_{2}=\{(1, i, j),(1, j, i) \mid 2 \leq$ $i<j \leq n\}$ is the set of 3 -cycles in $S_{n}$ moving 1, which ensures that the way of induction could be used to find the second largest eigenvalue of $C A G_{n}$. For this reason, we first need to consider the same problem for the graph $E A G_{n}$, and again, the main method depends on finding some suitable equitable partition to decompose the edge set of $E A G_{n}$.

For $1 \leq i, j \leq n$, we define

$$
X_{i}(j)=\left\{\tau \in A_{n} \mid \tau_{j}=i\right\} .
$$

For any fixed $i$ (resp. $j$ ), we see that $X_{i}(1), X_{i}(2), \ldots, X_{i}(n)\left(\right.$ resp. $X_{1}(j), X_{2}(j), \ldots$, $\left.X_{n}(j)\right)$ partition $A_{n}$. Now we shall verify that $\Pi: A_{n}=X_{i}(1) \cup X_{i}(2) \cup \cdots \cup X_{i}(n)$ is an equitable partition of $E A G_{n}$ for each $i$. For $\tau \in X_{i}(1)$, we have $N(\tau) \cap X_{i}(1)=\emptyset$ since $(\sigma \tau)_{1} \neq \tau_{1}=i$ for any $\sigma \in T_{2}$, and $N(\tau) \cap X_{i}(j)=\{(1, k, j) \tau \mid 2 \leq k \leq$ $n, k \neq j\}$ for $2 \leq j \leq n$. Thus each $\tau \in X_{i}(1)$ has no neighbors in $X_{i}(1)$ and $n-2$ neighbors in $X_{i}(j)$ for each $j(2 \leq j \leq n)$. For each fixed $j(2 \leq j \leq n)$, let $\tau \in X_{i}(j)$, we have $N(\tau) \cap X_{i}(1)=\{(1, j, k) \tau \mid 2 \leq k \leq n, k \neq j\}, N(\tau) \cap X_{i}(j)=$ $\{(1, k, l) \tau,(1, l, k) \tau \mid 2 \leq k<l \leq n, k, l \neq j\}$, and $N(\tau) \cap X_{i}(\ell)=\{(1, \ell, j) \tau\}$ for any $\ell \notin\{1, j\}$. This implies that $\tau$ has $n-2$ neighbors in $X_{i}(1),(n-2)(n-3)$ neighbors 
in $X_{i}(j)$, and one neighbor in $X_{i}(\ell)$ for each $\ell \notin\{1, j\}$. Thus the partition $\Pi$ is an equitable partition of $E A G_{n}$ with divisor matrix

$$
B_{\Pi}=\left[\begin{array}{ccccc}
0 & n-2 & n-2 & \cdots & n-2 \\
n-2 & (n-2)(n-3) & 1 & \cdots & 1 \\
n-2 & 1 & (n-2)(n-3) & \cdots & 1 \\
\vdots & \vdots & \vdots & \ddots & \vdots \\
n-2 & 1 & 1 & \cdots & (n-2)(n-3)
\end{array}\right] X_{i}(n)
$$

Then the characteristic polynomial of $B_{\Pi}$ is equal to

$$
\begin{aligned}
& \left|\lambda I_{n}-B_{\Pi}\right|=\left|\begin{array}{ccccc}
\lambda & -(n-2) & -(n-2) & \cdots & -(n-2) \\
-(n-2) & \lambda-(n-2)(n-3) & -1 & \cdots & -1 \\
-(n-2) & -1 & \lambda-(n-2)(n-3) & \cdots & -1 \\
\vdots & \vdots & \vdots & \ddots & \vdots \\
-(n-2) & -1 & -1 & \cdots & \lambda-(n-2)(n-3)
\end{array}\right| \\
& =(\lambda-(n-1)(n-2))\left|\begin{array}{ccccc}
1 & -(n-2) & -(n-2) & \cdots & -(n-2) \\
1 & \lambda-(n-2)(n-3) & -1 & \cdots & -1 \\
1 & -1 & \lambda-(n-2)(n-3) & \cdots & -1 \\
\vdots & \vdots & \vdots & \ddots & \vdots \\
1 & -1 & -1 & \cdots & \lambda-(n-2)(n-3)
\end{array}\right| \\
& =(\lambda-(n-1)(n-2))\left|\begin{array}{ccccc}
1 & 0 & 0 & \cdots & 0 \\
1 & \lambda-(n-2)(n-4) & n-3 & \cdots & n-3 \\
1 & n-3 & \lambda-(n-2)(n-4) & \cdots & n-3 \\
\vdots & \vdots & \vdots & \ddots & \vdots \\
1 & n-3 & n-3 & \cdots & \lambda-(n-2)(n-4)
\end{array}\right| \\
& =(\lambda-(n-1)(n-2))\left|\begin{array}{cccc}
\lambda-(n-2)(n-4) & n-3 & \cdots & n-3 \\
n-3 & \lambda-(n-2)(n-4) & \cdots & n-3 \\
\vdots & \vdots & \ddots & \vdots \\
n-3 & n-3 & \cdots & \lambda-(n-2)(n-4)
\end{array}\right| \\
& =(\lambda-(n-1)(n-2))\left|(\lambda-(n-2)(n-4)) I_{n-1}+(n-3)\left(J_{n-1}-I_{n-1}\right)\right| \\
& =(\lambda-(n-1)(n-2))\left(\lambda-\left(n^{2}-5 n+5\right)\right)^{n-2}(\lambda+(n-2)) \text {, }
\end{aligned}
$$

where $I_{n-1}$ and $J_{n-1}$ denote the identity matrix and all ones matrix of order $n-1$, respectively. By Lemma 2.1, we have

Lemma 3.1. Let $E A G_{n}$ be the extended alternating group graph with $n \geq 3$. Then $E A G_{n}$ has eigenvalues $(n-1)(n-2), n^{2}-5 n+5$ (with multiplicity at least $\left.n-2\right)$ and $2-n$. Moreover, if $f$ is an eigenvector of $E A G_{n}$ corresponding to any other eigenvalue, then for all $i, j \in\{1,2, \ldots, n\}$, we have

$$
\sum_{x \in X_{i}(j)} f(x)=0
$$

where $X_{i}(j)$ is defined in (3).

Now we give the second largest eigenvalue of $E A G_{n}$.

Theorem 3.2. Let $E A G_{n}$ be the extended alternating group graph with $n \geq 3$. Then the two largest eigenvalues of $E A G_{n}$ are $\lambda_{1}\left(E A G_{n}\right)=(n-1)(n-2)$ and $\lambda_{2}\left(E A G_{n}\right)=n^{2}-5 n+5$. 
Proof. Obviously, $\lambda_{1}\left(E A G_{n}\right)=(n-1)(n-2)$ because $E A G_{n}$ is $(n-1)(n-2)$ regular. Now we prove $\lambda_{2}\left(E A G_{n}\right)=n^{2}-5 n+5$ by induction on $n$. For $n=3$, we have $E A G_{3}=K_{3}$, which gives that $\lambda_{2}\left(E A G_{3}\right)=-1$, and the result follows. Now suppose $n \geq 4$ and assume that the result holds for $E A G_{n-1}$, i.e., $\lambda_{2}\left(E A G_{n-1}\right)=$ $(n-1)^{2}-5(n-1)+5$. Let $\lambda$ be an eigenvalue of $E A G_{n}$ that is not equal to $(n-1)(n-2), n^{2}-5 n+5$ or $2-n$, and let $f$ be any fixed eigenvector corresponding to $\lambda$. Then $f$ must sum to zero on $X_{i}(j)$ for all $1 \leq i, j \leq n$. Now we partition the vertex set of $E A G_{n}$ as $A_{n}=X_{1}(2) \cup X_{2}(2) \cup \cdots \cup X_{n}(2)$. As in the proof of Theorem 2.3, one can easily verify that the induce subgraph of $E A G_{n}$ on $X_{i}(2)$ is isomorphic to $E A G_{n-1}$ for each $i(1 \leq i \leq n)$. Let $E_{1}=\cup_{i=1}^{n} E\left(E A G_{n}\left[X_{i}(2)\right]\right)$ and $E_{2}=E\left(E A G_{n}\right) \backslash E_{1}$. We claim that $E_{2}$ is exactly the set of edges of the alternating group graph $A G_{n}=\operatorname{Cay}\left(A_{n}, T_{1}\right)$, where $T_{1}=\{(1,2, k),(1, k, 2) \mid 3 \leq k \leq n\}$. In fact, for any $\gamma \in X_{i}(2)$ and $\gamma^{\prime} \in X_{j}(2)(i \neq j)$, we have $\left\{\gamma, \gamma^{\prime}\right\} \in E\left(E A G_{n}\right)$ if and only if $\gamma^{\prime} \gamma^{-1} \in T_{2}$ (i.e., $\gamma^{\prime} \gamma^{-1}$ is a 3 -cycle moving 1 ), which is the case if and only $\gamma^{\prime} \gamma^{-1} \in T_{1}\left(\subseteq T_{2}\right)$ because $\gamma^{\prime} \gamma^{-1}$ must move 2 due to $i \neq j$. This implies that the edges in $E_{2}$ come from $T_{1}$. On the other hand, we see that $T_{1}$ can only be used to produce the edges in $E_{2}$ because each edge in $E_{1}$ comes from $T_{2} \backslash T_{1}$, i.e., the set of 3 -cycles in $T_{2}$ fixing 2 . Then

$$
\begin{aligned}
\lambda & =\frac{f^{T} A\left(E A G_{n}\right) f}{f^{T} f} \\
& =\frac{2 \sum_{\{x, y\} \in E\left(E A G_{n}\right)} f(x) f(y)}{\sum_{x \in A_{n}} f(x)^{2}} \\
& =\frac{2 \sum_{\{x, y\} \in E_{1}} f(x) f(y)}{\sum_{x \in A_{n}} f(x)^{2}}+\frac{2 \sum_{\{x, y\} \in E_{2}} f(x) f(y)}{\sum_{x \in A_{n}} f(x)^{2}} .
\end{aligned}
$$

For the first term, we have

$$
\begin{aligned}
\frac{2 \sum_{\{x, y\} \in E_{1}} f(x) f(y)}{\sum_{x \in A_{n}} f(x)^{2}} & =\frac{\sum_{i=1}^{n} 2 \sum_{\{x, y\} \in E\left(E A G_{n}\left[X_{i}(2)\right]\right)} f(x) f(y)}{\sum_{i=1}^{n} \sum_{x \in X_{i}(2)} f(x)^{2}} \\
& \leq \max _{1 \leq i \leq n} \frac{2 \sum_{\{x, y\} \in E\left(E A G_{n}\left[X_{i}(2)\right]\right)} f(x) f(y)}{\sum_{x \in X_{i}(2)} f(x)^{2}} \\
& \leq \lambda_{2}\left(E A G_{n-1}\right),
\end{aligned}
$$

where the last inequality follows from the fact $\sum_{x \in X_{i}(2)} f(x)=0$ for each $i$ according to Lemma 3.1. For the second term, since $f$ is orthogonal to the all ones vector $\mathbf{1}$, we have

$$
\frac{2 \sum_{\{x, y\} \in E_{2}} f(x) f(y)}{\sum_{x \in A_{n}} f(x)^{2}} \leq \max _{g \perp \mathbf{1}} \frac{g^{T} A\left(A G_{n}\right) g}{g^{T} g}=\lambda_{2}\left(A G_{n}\right) .
$$

Combining above two bounds, we conclude that

$$
\lambda \leq \lambda_{2}\left(E A G_{n-1}\right)+\lambda_{2}\left(A G_{n}\right)=(n-1)^{2}-5(n-1)+5+2 n-6=n^{2}-5 n+5
$$

by Theorem 2.3. Hence $\lambda_{2}\left(E A G_{n}\right)=n^{2}-5 n+5$, and our result follows. 
Now we focus on the complete alternating group graph $C A G_{n}=\operatorname{Cay}\left(A_{n}, T_{3}\right)$ where $T_{3}=\{(i, j, k),(i, k, j) \mid 1 \leq i<j<k \leq n\}$. Obviously, $C A G_{n}$ is a $2\left(\begin{array}{l}n \\ 3\end{array}\right)$ regular graph. As above, we shall verify that $\Pi: A_{n}=X_{i}(1) \cup X_{i}(2) \cup \cdots \cup X_{i}(n)$ is also an equitable partition of $C A G_{n}$ for each fixed $i$, where $X_{i}(j)$ is defined in (3). For each fixed $j(1 \leq j \leq n)$, let $\tau \in X_{i}(j)$. We have $N(\tau) \cap X_{i}(j)=$ $\{(k, l, m) \tau,(k, m, l) \tau \mid 1 \leq k<l<m \leq n, k, l, m \neq j\}$, and $N(\tau) \cap X_{i}(\ell)=$ $\{(\ell, j, k) \tau \mid 1 \leq k \leq n, k \neq \ell, j\}$ for each $\ell \neq j$. This implies that $\tau$ has $2\left(\begin{array}{c}n-1 \\ 3\end{array}\right)$ neighbors in $X_{i}(j)$, and $n-2$ neighbors in $X_{i}(\ell)$ for each $\ell \neq j$. Thus $\Pi$ is an equitable partition of $C A G_{n}$ with divisor matrix

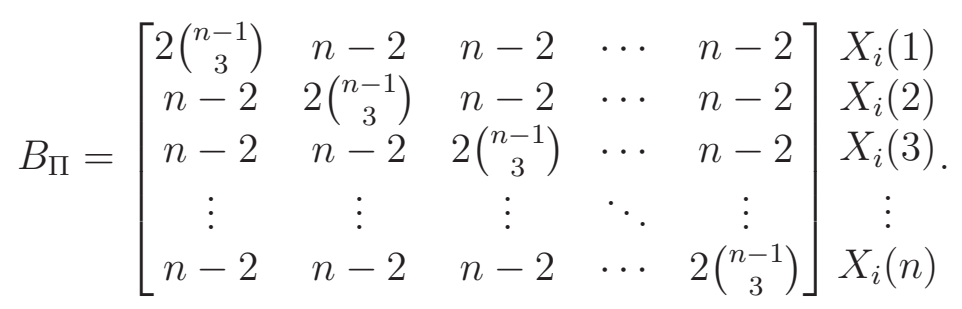

It is easy to see that $B_{\Pi}$ has eigenvalues $2\left(\begin{array}{l}n \\ 3\end{array}\right)$ and $\frac{1}{3} n(n-2)(n-4)$, where the first one is of multiplicity 1 having the all ones vector 1 as its eigenvector, and the second one is of multiplicity $n-1$ having each vector orthogonal to $\mathbf{1}$ as an eigenvector. Again by Lemma 2.1, we have

Lemma 3.3. Let $C A G_{n}$ be the complete alternating group graph with $n \geq 3$. Then $C A G_{n}$ has eigenvalues $2\left(\begin{array}{l}n \\ 3\end{array}\right)$ and $\frac{1}{3} n(n-2)(n-4)$ (with multiplicity at least $\left.n-1\right)$. Moreover, if $f$ is an eigenvector of $C A G_{n}$ corresponding to any other eigenvalue, then for all $i, j \in\{1,2, \ldots, n\}$, we have

$$
\sum_{x \in X_{i}(j)} f(x)=0
$$

where $X_{i}(j)$ is defined in (3).

Now we prove the main result of this section.

Theorem 3.4. Let $C A G_{n}$ be the complete alternating group graph with $n \geq 3$. Then the two largest eigenvalues of $C A G_{n}$ are $\lambda_{1}\left(C A G_{n}\right)=2\left(\begin{array}{l}n \\ 3\end{array}\right)$ and $\lambda_{2}\left(C A G_{n}\right)=$ $\frac{1}{3} n(n-2)(n-4)$.

Proof. Clearly, we have $\lambda_{1}\left(C A G_{n}\right)=2\left(\begin{array}{l}n \\ 3\end{array}\right)$ because $C A G_{n}$ is $2\left(\begin{array}{l}n \\ 3\end{array}\right)$-regular. We shall prove $\lambda_{2}\left(C A G_{n}\right)=\frac{1}{3} n(n-2)(n-4)$ by induction on $n$. For $n=3$, we have $\lambda_{2}\left(C A G_{3}\right)=\lambda_{2}\left(K_{3}\right)=-1$, as required. Now suppose $n \geq 4$. Assume that the result holds for $n-1$, i.e., $\lambda_{2}\left(C A G_{n-1}\right)=\frac{1}{3}(n-1)(n-3)(n-5)$. Let $\lambda$ be any eigenvalue of $C A G_{n}$ that is not equal to $2\left(\begin{array}{l}n \\ 3\end{array}\right)$ or $\frac{1}{3} n(n-2)(n-4)$, and pick any eigenvector $f$ of $C A G_{n}$ corresponding to $\lambda$. Again, the vector $f$ must sum to zero on $X_{i}(j)$ for all $i, j$ according to Lemma 3.3. We partition the vertex set of $C A G_{n}$ as $A_{n}=X_{1}(1) \cup X_{2}(1) \cup \cdots \cup X_{n}(1)$. Let $E_{1}=\left\{\{\tau, \sigma\} \in E\left(C A G_{n}\right) \mid \tau_{1} \neq \sigma_{1}\right\}$ and $E_{2}^{i}=\left\{\{\tau, \sigma\} \in E\left(C A G_{n}\right) \mid \tau_{1}=\sigma_{1}=i\right\}$ for $1 \leq i \leq n$. Then $E_{1} \cup E_{2}^{1} \cup E_{2}^{2} \cup \cdots \cup E_{2}^{n}$ is a partition of $E\left(C A G_{n}\right)$. As in the proof of Theorem 3.2, we see that $E_{1}$ is exactly the set of edges of the extended alternating group graph $E A G_{n}=\operatorname{Cay}\left(A_{n}, T_{2}\right)$ with 
$T_{2}=\{(1, k, l),(1, l, k) \mid 2 \leq k<l \leq n\}$, and for each $i, E_{2}^{i}$ is exactly the set of edges of the induced subgraph of $C A G_{n}$ on $X_{i}(1)$ which is also isomorphic to $C A G_{n-1}$. Then we have

$$
\begin{aligned}
\lambda & =\frac{f^{T} A\left(C A G_{n}\right) f}{f^{T} f} \\
& =\frac{2 \sum_{\{x, y\} \in E\left(C A G_{n}\right)} f(x) f(y)}{\sum_{x \in A_{n}} f(x)^{2}} \\
& =\frac{2 \sum_{\{x, y\} \in E_{1}} f(x) f(y)}{\sum_{x \in A_{n}} f(x)^{2}}+\frac{\sum_{i=1}^{n} 2 \sum_{\{x, y\} \in E_{2}^{i}} f(x) f(y)}{\sum_{x \in A_{n}} f(x)^{2}} .
\end{aligned}
$$

For the first term, since $f$ is orthogonal to the all ones vector $\mathbf{1}$, we have

$$
\frac{2 \sum_{\{x, y\} \in E_{1}} f(x) f(y)}{\sum_{x \in A_{n}} f(x)^{2}} \leq \max _{g \perp \mathbf{1}} \frac{g^{T} A\left(E A G_{n}\right) g}{g^{T} g}=\lambda_{2}\left(E A G_{n}\right) .
$$

For the second term, we have

$$
\begin{aligned}
\frac{\sum_{i=1}^{n} 2 \sum_{\{x, y\} \in E_{2}^{i}} f(x) f(y)}{\sum_{x \in A_{n}} f(x)^{2}} & =\frac{\sum_{i=1}^{n} 2 \sum_{\{x, y\} \in E\left(C A G_{n}\left[X_{i}(1)\right]\right)} f(x) f(y)}{\sum_{i=1}^{n} \sum_{x \in X_{i}(1)} f(x)^{2}} \\
& \leq \max _{1 \leq i \leq n} \frac{2 \sum_{\{x, y\} \in E\left(C A G_{n}\left[X_{i}(1)\right]\right)} f(x) f(y)}{\sum_{x \in X_{i}(1)} f(x)^{2}} \\
& \leq \lambda_{2}\left(C A G_{n-1}\right),
\end{aligned}
$$

where the last inequality follows from the fact $\sum_{x \in X_{i}(1)} f(x)=0$ for each $i$ according to Lemma 3.1. Combining above two bounds, we conclude that

$\lambda \leq \lambda_{2}\left(E A G_{n}\right)+\lambda_{2}\left(C A G_{n-1}\right)=n^{2}-5 n+5+\frac{1}{3}(n-1)(n-3)(n-5)=\frac{1}{3} n(n-2)(n-4)$

by Theorem 3.2. Hence $\lambda_{2}\left(E A G_{n}\right)=\frac{1}{3} n(n-2)(n-4)$, as required.

At the end of this paper, we pose the following problem.

Problem 1. How to determine all distinct eigenvalues (with multiplicities) of $A G_{n}$, $E A G_{n}$ and $C A G_{n}$ ?

\section{Acknowledgments}

The authors are grateful to the anonymous referees for their useful and constructive comments, which have considerably improved the presentation of this paper.

\section{References}

[1] D. Aldous, https://www. stat. berkeley . edu/users/aldous/Research/OP/index.html. 
[2] P. Caputo, T.M. Liggett, T. Richthammer, Proof of Aldous' spectral gap conjecture, J. Amer. Math. Soc. 23(3) (2010) 831-851.

[3] F. Cesi, Cayley graphs on the symmetric group generated by initial reversals have unit spectral gap, Electron. J. Combin. 16(1) (2009) \#N29.

[4] F. Cesi, On the eigenvalues of Cayley graphs on the symmetric group generated by a complete multipartite set of transpositions, J. Algebraic Combin. 32(2) (2010) $155-185$.

[5] J.M. Chang, J.S. Yang, Panconnectivity, fault tolerant Hamiltonicity and Hamiltonian connectivity in alternating group graphs, Networks 44 (2004) 302-310.

[6] J.M. Chang, J.S. Yang, Fault-tolerant cycle-embedding in alternating group graphs, Appl. Math. Comput. 197 (2008) 760-767.

[7] Y.K. Cheng, T. Lau, K.B. Wong, Cayley graph on symmetric group generated by elements fixing $k$ points, Linear Algebra Appl. 471 (2015) 405-426.

[8] F. Chung, J. Tobin, The spectral gap of graphs arising from substring reversals, Electron. J. Combin. 23(3) (2017) \#P3.4.

[9] D.M. Cvetković, P. Rowlinson, S.K. Simić, An Introduction to the Theory of Graph Spectra, Cambridge Univ. Press, Cambridge, 2010.

[10] P. Diaconis, M. Shahshahani, Generating a random permutation with random transpositions, Z. Wahrscheinlichkeitstheor. Verw. Geb. 57(2) (1981) 159-179.

[11] L. Flatto, A.M. Odlyzko, D.B. Wales, Random shuffles and group representations, Ann. Probab. 13(1) (1985) 154-178.

[12] J. Friedman, On Cayley graphs on the symmetric group generated by transpositions, Combinatorica 20(4) (2000) 505-519.

[13] C.D. Godsil, G. Royle, Algebraic Graph Theory, in: Graduate Texts in Mathematics, vol. 207, Springer, New York, 2001.

[14] X.Y. Huang, Q.X. Huang, Automorphism group of the complete alternating group graph, Appl. Math. Comput. 314 (2017) 58-64.

[15] J.S. Jwo, S. Lakshmivarahan, S.K. Dhall, A new class of interconnection networks based on the alternating group, Networks 23 (1993) 315-326.

[16] M. Suzuki, Group Theory I, Springer, New York, 1982.

[17] J.X. Zhou, The automorphism group of the alternating group graph, Appl. Math. Lett. 24 (2011) 229-231. 